\title{
Efeito do ácido indolbutírico no enraizamento de estacas de ramos semilenhosos de pessegueiro(1)
}

\author{
Mauro Brasil Dias Tofanelli(2), Nilton Nagib Jorge Chalfun ${ }^{(3)}$, Alexandre Hoffmann ${ }^{(4)}$ e Antônio Chalfun Júnior(5)
}

\begin{abstract}
Resumo - Neste trabalho objetivou-se estudar o efeito do ácido indolbutírico (AIB) na formação de raízes adventícias em estacas semilenhosas de nove cultivares-copa (Arlequim, Aurora, Biuti, Diamante, Momo, Ouromel, Pérola de Mairinque, Premier e Tropical) e dois porta-enxertos (Okinawa e R-15-2) de pessegueiro. O experimento foi realizado na Universidade Federal de Lavras, Lavras, MG, em nov./dez. de 1997. As concentrações de AIB utilizadas foram de 0, 1.000, 2.000 e $3.000 \mathrm{mg} \mathrm{L}^{-1}$, e as estacas tiveram a base imersa em solução durante cinco segundos. O AIB influenciou no enraizamento, na formação de calo e no número de raízes. As maiores porcentagens de enraizamento foram observadas nas cultivares Pérola de Mairinque (56,62\%) e Tropical (55,04\%), ambas ocorrendo na concentração de $3.000 \mathrm{mg} \mathrm{L}^{-1} \mathrm{de}$ AIB.
\end{abstract}

Termos para indexação: Prunus persica, raízes adventícias, auxina, propagação vegetal.

\section{Effect of indolebutyric acid on rooting ability of semihardwood stem cuttings of peach}

\begin{abstract}
This work had as objective to evaluate the effect of indolebutyric acid (IBA) on the rooting of semihardwood cuttings of nine cultivars (Arlequim, Aurora, Biuti, Diamante, Momo, Ouromel, Pérola de Mairinque, Premier and Tropical) and of two rootstocks (Okinawa and R-15-2) of peach. The experiment was set up at the Universidade Federal de Lavras, Lavras, MG, Brazil, in Nov./Dec. 1997. The IBA concentrations were $0 ; 1,000 ; 2,000$ and 3,000 $\mathrm{mg} \mathrm{L}^{-1}$, and the cutting base was dipped in the solution for five seconds. IBA influence was detected on rooting, on callus formation and root number. The highest percentage of rooting occurred in cultivars Pérola de Mairinque $(56.62 \%)$ and Tropical (55.04\%), both at the concentration of $3,000 \mathrm{mg} \mathrm{L}^{-1}$ of IBA.
\end{abstract}

Index terms: Prunus persica, adventitious roots, auxin, plant propagation.

\section{Introdução}

O pessegueiro (Prunus persica (L.) Batsch) é uma das principais frutíferas de clima temperado cultivadas na região Sul do Brasil. A produção vem crescendo ao longo dos anos, passando de $77.340 \mathrm{t} \mathrm{em}$ 1991 para 112.040 t em 1998 (Agrianual, 1999).

(1) Aceito para publicação em 24 de outubro de 2001 .

(2) Universidade Estadual Paulista, Fac. de Ciências Agrárias, Caixa Postal 237, CEP 18603-970 Botucatu, SP. E-mail maurobdt@fca.unesp.br

(3) Universidade Federal de Lavras, Caixa Postal 37 , CEP 37200-000 Lavras, MG. E-mail: nchalfun@ufla.br

(4) Embrapa-Centro Nacional de Pesquisa de Uva e Vinho, Caixa Postal 177, CEP 95200-000 Vacaria, RS. E-mail: hoffmann@cnpuv.embrapa.br

(5) Wageningen University, P.O. Box 16 Droevendaalsesteeg, 1, 6700 AA, Wageningen, The Netherlands. E-mail A.Chalfun-Junior@plant.wa-ur.nl
O aumento da produção é acompanhado por novas tecnologias de cultivo, que possibilitam aumentar a produtividade e a rentabilidade do negócio; mesmo assim, a ampliação dos pomares desta espécie tornar-se-ia necessária para alcançar produções ainda mais elevadas. Assim, novas alternativas de propagação poderiam incentivar o aumento de áreas plantadas desta frutífera.

A propagação do pessegueiro no Brasil é, basicamente, realizada através da enxertia de borbulhas sobre porta-enxertos provenientes de sementes (Fachinello et al., 1982, 1984; Chalfun et al., 1994; Chalfun \& Hoffmann, 1997; Tonietto et al., 1997). Neste método de propagação, pode haver perda de características interessantes da planta matriz aos porta-enxertos (Chalfun et al., 1994), gerando indivíduos que variam morfológica e fisiologicamente entre si, além de a vida útil produtiva da copa ser limitada pela longevidade do material utilizado como por- 
ta-enxerto (Manual..., 1988; Kersten, 1991; Pasinatto et al., 1998).

O uso de estacas para a produção de mudas de pessegueiro pode ser uma maneira de evitar estes inconvenientes e possibilitar a produção rápida, simples e de baixo custo de um maior número de mudas em um menor espaço de tempo (Sharma \& Aier, 1989; Mesén et al., 1997). Autores como Couvillon \& Erez (1980) e Avery \& Beyl (1991) já destacavam o uso da estaquia como uma forma de produção de mudas em massa para pomares adensados.

Apesar de o método de propagação por estaca ser bastante interessante, não tem sido uma alternativa viável para algumas espécies, em face de alguns entraves, como a baixa capacidade de enraizar estacas, e da carência de informações de pomares formados com mudas oriundas de estacas, como é o caso do pessegueiro no Brasil. Algumas técnicas são utilizadas para tentar maximizar o porcentual de enraizamento de estacas, e entre as mais utilizadas destaca-se a aplicação exógena de hormônios sintéticos de crescimento da planta. $\mathrm{O}$ ácido indolbutírico (AIB) é um dos mais empregados e mais eficientes (Dunn et al., 1996; Tonietto et al., 1997; Dutra et al., 1998), por ser fotoestável e ser imune à ação biológica (Hoffmann et al., 1996; Ono \& Rodrigues, 1996).

Tonietto et al. (1997) observaram, na cultivar Diamante, que o porcentual de estacas enraizadas aumentou até a concentração de $1.752 \mathrm{mg} \mathrm{L}^{-1}$ de AIB. Couvillon \& Erez (1980) obtiveram 100\% de enraizamento tratando estacas semilenhosas de pessegueiro da seleção $1.372 \mathrm{com} 2.500 \mathrm{mg} \mathrm{L}^{-1}$ de AIB. Já Kaundal et al. (1993) observaram que os maiores porcentuais de enraizamento foram obtidos com a concentração de $500 \mathrm{mg} \mathrm{L}^{-1}$ de AIB em estacas das cultivares Flordasun (42,2\%), Shan-i-Punjab (38,8\%) e Florda Red (41,2\%) e de $1.500 \mathrm{mg} \mathrm{L}^{-1}$ de AIB em estacas da cultivar Sharbati (38,7\%).

O objetivo deste trabalho foi avaliar o efeito de diferentes concentrações de ácido indolbutírico no enraizamento de estacas de ramos semilenhosos de cultivares de pessegueiro.

\section{Material e Métodos}

Estacas de pessegueiro foram coletadas em Lavras, MG, em final de novembro e início de dezembro de 1997, de plantas com seis a sete anos de idade existentes na coleção de cultivares da Universidade Federal de Lavras. Foram estudadas no experimento as cultivares-copa Arlequim, Aurora, Biuti, Diamante, Momo, Ouromel, Pérola de Mairinque, Premier e Tropical e os porta-enxertos Okinawa e R-15-2. As estacas foram preparadas com comprimento de 15 a $20 \mathrm{~cm}$ e diâmetro de 5 a $7 \mathrm{~mm}$, mantendo-se duas folhas seccionadas ao meio por estaca.

Para o preparo da solução de AIB $\left(\mathrm{C}_{12} \mathrm{H}_{13} \mathrm{NO}_{2}\right)$, foi feita uma pré-diluição do regulador (forma de pó) com algumas gotas de $\mathrm{NaOH} 0,5 \mathrm{~N}$, sendo em seguida completado o volume final com água destilada.

O substrato utilizado foi a areia lavada, colocada em sacos de polietileno preto medindo $10 \mathrm{~cm}$ de diâmetro por $20 \mathrm{~cm}$ de altura. Após o plantio, as estacas foram colocadas em casa de vegetação com sistema de nebulização intermitente, onde permaneceram por um período de 60 dias, com temperatura variando entre 25 e $33^{\circ} \mathrm{C}$ e umidade entre 80 e $90 \%$.

O delineamento experimental adotado foi o inteiramente casualizado. Adotou-se um esquema fatorial $11 \times 4$, envolvendo 11 cultivares e quatro dosagens de AIB (0, $1.000,2.000$ e $3.000 \mathrm{mg} \mathrm{L}^{-1}$ ), com quatro repetições e dez estacas por repetição em cada parcela.

Após 60 dias do plantio, foram avaliadas as porcentagens de estacas enraizadas, estacas com calo e número médio de raízes por estaca enraizada em cada tratamento. Os resultados obtidos foram submetidos à análise de variância, e as médias, comparadas pelo teste de Duncan a 5\% de probabilidade. As interações significativas foram submetidas à análise de regressão, para o estudo do efeito do AIB. Efetuou-se transformação de dados segundo a equação arco seno $(\mathrm{x} / 100)^{0,5}$ para as variáveis cujos valores eram em porcentagem, e $(\mathrm{x}+1)^{0,5}$ para a variável número de raízes.

\section{Resultados e Discussão}

A cultivar Tropical foi superior às demais quanto ao enraizamento $(28,22 \%)$, embora tenha sido estatisticamente igual às cultivares Pérola de Mairinque $(26,43 \%)$ e Biuti (17,56\%) (Tabela 1). Respostas diferenciadas entre cultivares, quanto à capacidade de formar raízes adventícias em estacas, têm sido relatadas em vários trabalhos, e umas das principais explicações para este comportamento é a constituição genética das cultivares que resultaria em um potencial de enraizamento diferenciado entre elas (Hoffmann et al., 1995; Dutra et al., 1998). O efeito significativo do AIB no enraizamento demonstrou 
Tabela 1. Porcentagens de enraizamento e de formação de calo e número de raízes em estacas semilenhosas de cultivares de pessegueiro, em quatro concentrações de ácido indolbutírico. Lavras, Ufla, 2001(1).

\begin{tabular}{|c|c|c|c|c|c|c|c|}
\hline Cultivar & 0 & 1.000 & $\begin{array}{c}2.000 \\
\left.L^{-1}\right)\end{array}$ & 3.000 & Média & Equação & $\mathrm{r}^{2}$ \\
\hline \multicolumn{8}{|c|}{ Enraizamento (\%) } \\
\hline Arlequim & $0,00 \mathrm{aB}$ & $12,23 \mathrm{abcA}$ & $2,57 \mathrm{dAB}$ & $0,00 \mathrm{eB}$ & $1,67 \mathrm{f}$ & $Y=1,6875+0,0211387 X-0,00000742 X^{2}$ & 0,80 \\
\hline Aurora & $1,58 \mathrm{aB}$ & $13,89 \mathrm{abcAB}$ & $36,61 \mathrm{aA}$ & $19,48 \mathrm{bcdA}$ & $15,44 \mathrm{bcd}$ & $\mathrm{Y}=5,882277+0,0264879 \mathrm{X}-0,00000642 \mathrm{X}^{2}$ & 0,92 \\
\hline Biuti & $0,00 \mathrm{aB}$ & $24,04 \mathrm{abA}$ & $39,52 \mathrm{aA}$ & $26,22 \mathrm{bcA}$ & $17,56 a b c$ & $\mathrm{Y}=0,101856+0,0383299 \mathrm{X}-0,00000938 \mathrm{X}^{2}$ & 0,99 \\
\hline Diamante & $0,00 \mathrm{aA}$ & 4,99bcdA & $7,48 \mathrm{bcdA}$ & $2,90 \mathrm{deA}$ & 2,81ef & - & - \\
\hline Momo & $0,00 \mathrm{aA}$ & $0,00 \mathrm{dA}$ & $0,65 \mathrm{dA}$ & $6,20 \mathrm{cde} A$ & $0,69 \mathrm{f}$ & $Y=0,0002+0,0047859 X$ & 0,83 \\
\hline Ouromel & $0,00 \mathrm{aB}$ & $0,00 \mathrm{~dB}$ & $23,71 \mathrm{abA}$ & $32,05 \mathrm{bA}$ & $7,51 \mathrm{de}$ & $\mathrm{Y}=0,0001+0,0132588 \mathrm{X}$ & 0,86 \\
\hline Okinawa & $0,00 \mathrm{aB}$ & $15,58 \mathrm{abcA}$ & $21,08 \mathrm{abcA}$ & $18,67 \mathrm{bcdA}$ & $10,65 \mathrm{~cd}$ & $Y=0,667975+0,0268176 X-0,00000624 X^{2}$ & 0,98 \\
\hline Per. Mair. & $0,00 \mathrm{aC}$ & $24,04 \mathrm{abB}$ & $43,57 \mathrm{aAB}$ & $63,92 \mathrm{aA}$ & $26,43 a b$ & $\mathrm{Y}=5,258168+0,0171196 \mathrm{X}$ & 0,94 \\
\hline Premier & $0,65 \mathrm{aAB}$ & $2,08 \mathrm{cdAB}$ & $9,44 \mathrm{bcdA}$ & $0,00 \mathrm{eA}$ & $1,80 \mathrm{ef}$ & $\mathrm{Y}=2,94002+0,0157652 \mathrm{X}-0,0000054 \mathrm{X}^{2}$ & 0,68 \\
\hline $\mathrm{R}-15-2$ & $0,00 \mathrm{aB}$ & $16,75 \mathrm{abcA}$ & $3,81 \mathrm{cdAB}$ & $0,65 \mathrm{eB}$ & $3,02 \mathrm{ef}$ & $Y=2,167157+0,0231935 X-0,0000077 X^{2}$ & 0,72 \\
\hline Tropical & $0,65 \mathrm{aC}$ & $26,22 \mathrm{aB}$ & $42,37 \mathrm{aAB}$ & $62,66 \mathrm{aA}$ & $28,22 \mathrm{a}$ & $\mathrm{Y}=9,140934+0,0152992 \mathrm{X}$ & 0,94 \\
\hline Média & $0,68 \mathrm{C}$ & $9,78 \mathrm{~B}$ & $17,67 \mathrm{~A}$ & $15,08 \mathrm{~A}$ & & & \\
\hline \multicolumn{8}{|c|}{ Formação de calos (\%) } \\
\hline Arlequim & $0,00 \mathrm{cA}$ & $0,00 \mathrm{dA}$ & $0,00 \mathrm{bA}$ & $0,00 \mathrm{bA}$ & $0,00 \mathrm{c}$ & - & - \\
\hline Aurora & $21,60 \mathrm{bA}$ & $1,59 \mathrm{cdB}$ & $0,00 \mathrm{bB}$ & $1,59 \mathrm{abB}$ & $3,35 \mathrm{~b}$ & $Y=27,761654-0,0276366 X+0,00000692 X^{2}$ & 0,99 \\
\hline Biuti & $18,76 \mathrm{bA}$ & $7,47 \mathrm{bcAB}$ & $0,65 \mathrm{bB}$ & $2,57 \mathrm{abB}$ & $5,72 \mathrm{~b}$ & $\mathrm{Y}=22,927507-0,060597 \mathrm{X}$ & 0,74 \\
\hline Diamante & $55,13 \mathrm{aA}$ & $23,71 \mathrm{abB}$ & $18,67 \mathrm{aB}$ & $0,00 \mathrm{bC}$ & $18,77 \mathrm{a}$ & $Y=47,779958-0,014738 X$ & 0,93 \\
\hline Momo & $0,00 \mathrm{cA}$ & $0,00 \mathrm{dA}$ & $0,65 \mathrm{bA}$ & $0,00 \mathrm{bA}$ & $0,04 \mathrm{c}$ & - & - \\
\hline Ouromel & $0,00 \mathrm{cA}$ & $0,00 \mathrm{dA}$ & $0,00 \mathrm{bA}$ & $0,00 \mathrm{bA}$ & $0,00 \mathrm{c}$ & - & - \\
\hline Okinawa & $0,00 \mathrm{cA}$ & $0,00 \mathrm{dA}$ & $0,00 \mathrm{bA}$ & $0,00 \mathrm{bA}$ & $0,00 \mathrm{c}$ & - & - \\
\hline Per. Mair. & $33,50 \mathrm{abA} 7$ & $6,65 \mathrm{cdB}$ & $0,00 \mathrm{bC}$ & $1,34 \mathrm{abBC}$ & $6,06 \mathrm{~b}$ & $Y=36,2144553-0,030457 X+0,00000678 X^{2}$ & 0,98 \\
\hline Premier & $0,00 \mathrm{cA}$ & $0,00 \mathrm{dA}$ & $0,00 \mathrm{bA}$ & $0,00 \mathrm{bA}$ & $0,00 \mathrm{c}$ & - & - \\
\hline $\mathrm{R}-15-2$ & $0,00 \mathrm{cA}$ & $3,81 \mathrm{cdA}$ & $5,28 \mathrm{abA}$ & $5,28 \mathrm{abA}$ & $2,70 \mathrm{~b}$ & - & - \\
\hline Tropical & $48,50 \mathrm{aA} 9$ & $44,48 \mathrm{aA}$ & $14,92 \mathrm{aB}$ & $9,44 \mathrm{aB}$ & $27,55 \mathrm{a}$ & $Y=46,360210-0,0098011 X$ & 0,91 \\
\hline Média & $8,02 \mathrm{~A}$ & $3,60 \mathrm{~B}$ & $1,26 \mathrm{C}$ & $0,74 \mathrm{C}$ & & & \\
\hline \multicolumn{8}{|c|}{ Número de raízes } \\
\hline Arlequim & $0,00 \mathrm{aA}$ & $3,79 \mathrm{aA}$ & $3,40 \mathrm{cdA}$ & $0,00 \mathrm{dA}$ & $1,47 \mathrm{c}$ & - & - \\
\hline Aurora & $0,83 \mathrm{aB}$ & $3,43 \mathrm{aB}$ & $23,85 \mathrm{aA}$ & $19,00 \mathrm{abcAB}$ & $9,43 \mathrm{ab}$ & $\mathrm{Y}=1,393527+0,012242 \mathrm{X}$ & 0,84 \\
\hline Biuti & $0,00 \mathrm{aC}$ & $7,83 \mathrm{aB}$ & $27,97 \mathrm{aA}$ & $17,14 \mathrm{bcdAB}$ & $10,58 \mathrm{ab}$ & $\mathrm{Y}=0,801282+0,0035404 \mathrm{X}-0,00000077 \mathrm{X}^{2}$ & 0,99 \\
\hline Diamante & $0,00 \mathrm{aB}$ & $3,66 \mathrm{aAB}$ & $12,08 \mathrm{abcdA}$ & $6,99 \mathrm{bcdAB}$ & $4,76 \mathrm{bc}$ & $\mathrm{Y}=1,359668+0,0006938 \mathrm{X}$ & 0,72 \\
\hline Momo & $0,00 \mathrm{aA}$ & $0,00 \mathrm{aA}$ & $1,25 \mathrm{dA}$ & $4,51 \mathrm{cdA}$ & $1,14 \mathrm{c}$ & - & - \\
\hline Ouromel & $0,00 \mathrm{aB}$ & $0,00 \mathrm{aB}$ & $18,40 \mathrm{abA}$ & $29,17 \mathrm{aA}$ & $7,85 \mathrm{ab}$ & $Y=0,441624+0,016887 X$ & 0,86 \\
\hline Okinawa & $0,00 \mathrm{aC}$ & $2,71 \mathrm{aBC}$ & $12,97 \mathrm{abcAB}$ & $16,67 \mathrm{abcA}$ & $3,38 \mathrm{ab}$ & $Y=1,003933+0,011422 X$ & 0,98 \\
\hline Per. Mair. & $0,00 \mathrm{aC}$ & $6,01 \mathrm{aBC}$ & $16,83 \mathrm{abcAB}$ & $38,79 \mathrm{aA}$ & $11,56 \mathrm{a}$ & $\mathrm{Y}=0,920109+0,0017498 \mathrm{X}$ & 0,99 \\
\hline Premier & $0,22 \mathrm{aA}$ & $1,25 \mathrm{aA}$ & $6,51 \mathrm{bcdA}$ & $0,00 \mathrm{dA}$ & $1,52 \mathrm{c}$ & - & - \\
\hline $\mathrm{R}-15-2$ & $0,00 \mathrm{aA}$ & $4,23 \mathrm{aA}$ & $1,15 \mathrm{dA}$ & $0,40 \mathrm{dA}$ & $1,20 \mathrm{c}$ & - & - \\
\hline Tropical & $0,22 \mathrm{aB}$ & $6,91 \mathrm{aAB}$ & $16,98 \mathrm{abcA}$ & $22,42 \mathrm{abA}$ & $9,55 \mathrm{ab}$ & $Y=1,353557+0,0012635 X$ & 0,96 \\
\hline Média & $0,10 \mathrm{C}$ & $3,22 \mathrm{~B}$ & $11,18 \mathrm{~A}$ & $10,89 \mathrm{~A}$ & & & \\
\hline
\end{tabular}


que as concentrações de 2.000 e $3.000 \mathrm{mg} \mathrm{L}^{-1}$ foram as que proporcionaram os melhores resultados (Tabela 1). A análise de regressão polinomial demonstrou que as cultivares Momo, Pérola de Mairinque, Ouromel e Tropical obtiveram incrementos lineares no porcentual de enraizamento à medida que se aumentava a concentração de AIB, e alcançaram os melhores resultados $(11,94 \% ; 56,62 \% ; 35,79 \%$ e $55,04 \%$ nas respectivas cultivares), com $3.000 \mathrm{mg} \mathrm{L}^{-1}$ (Tabela 1). As cultivares Arlequim, Aurora, Biuti, Premier, Okinawa e R-15-2 demonstraram efeito quadrático do AIB no enraizamento das estacas, nas quais os respectivos porcentuais máximos foram de $16,74 \%$ com $1.424,44 \mathrm{mg} \mathrm{L}^{-1} ; 33,20 \%$ com $2.062,92 \mathrm{mg} \mathrm{L}^{-1} ; 39,26 \% \operatorname{com} 2.043,17 \mathrm{mg} \mathrm{L}^{-1} ; 14,45 \%$ com 1.459,74 mg L-1;29,48\% com 2.148,85 mg L-1; $19,63 \%$ com $1.506,07 \mathrm{mg} \mathrm{L}^{-1}$. A cultivar Diamante não obteve regressão significativa a $5 \%$ de probabilidade. Fachinello et al. (1984) estudaram o efeito do AIB na porcentagem de estacas lenhosas enraizadas de pessegueiro e constataram que o máximo de enraizamento na cultivar Capdeboscq $(68,9 \%)$ ocorreu com $2.790 \mathrm{mg} \mathrm{L}^{-1}$ de AIB, e na cultivar Diamante (54,0\%) ocorreu com $2.580 \mathrm{mg} \mathrm{L}^{-1}$. Segundo Kersten et al. (1994), a concentração ideal do hormônio sintético da planta na solução de aplicação depende do balanço hormonal entre auxinas endógenas e exógenas. Além disso, de acordo com Garrido et al. (1998), a diferença de sensibilidade à auxina das estacas entre cultivares pode promover mudanças no processo de enraizamento de estacas.

As cultivares Tropical $(27,55 \%)$ e Diamante $(18,77 \%)$ foram superiores às demais quanto ao porcentual de formação de calo nas estacas (Tabela 1). Pelos resultados obtidos nas variáveis enraizamento e formação de calo, observa-se que, das cultivares que apresentaram maior porcentual de estacas com calo (Tropical e Diamante), somente a Tropical apresentou maior enraizamento. Sendo assim, no presente trabalho, não houve relação entre enraizamento e formação de calo, o que pode ser comprovado pela correlação linear não-significativa apresentada entre as duas variáveis (Tabela 2). Esse comportamento pode ocorrer porque esses dois processos são independentes, e podem ser, ou não, influenciados pelos mesmos fatores (Fachinello et al., 1995). Além disso, a formação de calo pode, ou não, resultar em formação de raízes. Leonel et al. (1994) mencionaram que a formação de calo pode ser o primeiro passo para a formação do sistema radicular. Por outro lado, Pacheco et al. (1998) demonstraram que a formação de raízes não ocorreu diretamente do calo no enraizamento da videira Muscadínia (Vitis rotundifolia Michx.) usando alporquia. Já Rossal et al. (1997) relataram que a formação de calo em estacas de laranjeira (Citrus sinensis (L.) Osbeck) da cultivar Valência pode ter ocorrido em virtude do equilíbrio favorável entre auxinas e citocininas endógenas, o que não deve ter acontecido no presente trabalho. O AIB influenciou negativamente nesta variável, pois houve diminuição linear dos valores nas cultivares Biuti, Diamante e Tropical até a concentração de $3.000 \mathrm{mg} \mathrm{L}^{-1}$, e nas cultivares Aurora e Pérola de Mairinque houve diminuição quadrática com leve aumento das porcentagens a partir de $1.996,86 \mathrm{mg} \mathrm{L}^{-1}$ e $2.246,09 \mathrm{mg} \mathrm{L}^{-1}$, respectivamente (Tabela 1). Isto pode ter sido causado pelo desequilíbrio hormonal provocado pela aplicação exógena do AIB, desfavorecendo a formação de calo, já que a testemunha apresentou o maior porcentual de estacas com calo. Mattiuz \& Fachinello (1996) também observaram maior formação de calo no tratamento $0 \mathrm{mg} \mathrm{L}^{-1}$ de AIB em estacas de kiwi (Actinidia deliciosa (A. Chev.) C. F. Liang e A. R. Ferguson var. Deliciosa) das cultivares Tomuri e Bruno. As cultivares Arlequim, Momo, Premier, Ouromel, Okinawa e R-15-2 não obtiveram regressões significativas a 5\% de probabilidade. Embora não tenha obtido correlação significativa entre enraizamento e formação de calo, houve tendência de aumento de enraizamento e de diminuição na formação de calo com aplicação de AIB naquelas cultivares que apre-

Tabela 2. Coeficientes de correlação linear simples entre as variáveis enraizamento, formação de calos e número de raízes em estacas semilenhosas de pessegueiro. Lavras, Ufla, 2001.

\begin{tabular}{lc}
\hline Variáveis & $\begin{array}{c}\text { Coeficientes de } \\
\text { correlação }\end{array}$ \\
\hline Enraizamento x calo & $-0,0314874^{\text {ns }}$ \\
Enraizamento x número de raízes & $0,7586712 * *$ \\
Calo x número de raízes & $-0,1482113 *$ \\
\hline
\end{tabular}

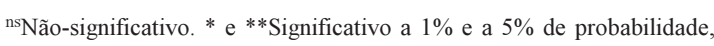
respectivamente 
sentaram regressões significativas (Tabela 1). Isto sugere que estes dois fenômenos seriam antagônicos, pois poderiam estar competindo por assimilados.

O maior número de raízes foi obtido na cultivar Pérola de Mairinque, embora esta tenha sido estatisticamente superior apenas às cultivares Arlequim, Diamante, Momo, Premier e R-15-2. As concentrações de AIB que promoveram maior número de raízes foram 2.000 e $3.000 \mathrm{mg} \mathrm{L}^{-1}$ (Tabela 1). As cultivares Aurora, Diamante, Pérola de Mairinque, Okinawa, Ouromel e Tropical demonstraram aumento no número de raízes por estaca enraizada até a concentração de $3.000 \mathrm{mg} \mathrm{L}^{-1}$ de AIB e a cultivar Biuti obteve máximo de 4,87 raízes por estaca enraizada, com 2.298,96 mg L-1. Tonietto et al. (1997) também obtiveram efeito significativo do AIB sobre o número de raízes, no qual houve incremento até a concentração de $1.791 \mathrm{mg} \mathrm{L}^{-1}$ de AIB. Correlação significativa pôde ser observada entre enraizamento e número de raízes (Tabela 2). Houve tendência de aumento dos porcentuais de enraizamento e número de raízes com a aplicação do AIB (Tabela 1).

\section{Conclusões}

1. O enraizamento, formação de calo e número de raízes em estacas semilenhosas de pessegueiro são afetados pela cultivar e pelo ácido indolbutírico (AIB).

2. O AIB é eficiente na promoção do enraizamento de estacas semilenhosas das cultivares de pessegueiro, e as melhores concentrações são $2.000 \mathrm{e}$ $3.000 \mathrm{mg} \mathrm{L}^{-1}$ de AIB.

3. As cultivares Tropical e Pérola de Mairinque são as que apresentam melhores respostas com $3.000 \mathrm{mg} \mathrm{L}^{-1}$ de AIB, indicando, assim, o potencial da técnica para propagação comercial.

4. O AIB não favorece a formação de calo, mas favorece o aumento do número de raízes em algumas cultivares.

\section{Referências}

AGRIANUAL 2000: anuário da agricultura brasileira. São Paulo: FNP Consultoria, 1999. 546 p.
AVERY, J. D.; BEYL, C. B. Propagation of peach cuttings using foam cubes. HortScience, Alexandria, v. 26, n. 9, p. 1152-1154, Sept. 1991.

CHALFUN, N. N. J.; HOFFMANN, A. Propagação do pessegueiro e da ameixeira. Informe Agropecuário, Belo Horizonte, v. 18, n. 189, p. 23-29, 1997.

CHALFUN, N. N. J.; PASQUAL, M.; RAMOS, J. D.; LIMA, P. C.; CHALFUN JÚNIOR, A.; SILVA, T. das G. Efeito do anelamento e diferentes dosagens do ácido indolbutírico na propagação de estacas caulinares do pessegueiro "Okinawa". Revista Brasileira de Fruticultura, Cruz das Almas, v. 16, n. 1, p. 119-126, 1994.

COUVILlON, G. A.; EREZ, A. Rooting, survival and development of several peach cultivars propagated from semihardwood cuttings. HortScience, Alexandria, v. 15, n. 1, p. 41-43, Feb. 1980.

DUNN, D. E.; COLE, J. C.; SMITH, M. W. Position of cut, bud retention and auxins influence rooting of Pistacia chinensis. Scientia Horticulturae, Amsterdam, v. 67, n. 1/2, p. 105-110, Nov. 1996.

DUTRA, L. F.; TONIETTO, A.; KERSTEN, E. Efeito da aplicação de ethefon em ameixeira (Prunus salicina Lindl) e do IBA no enraizamento de suas estacas. Scientia Agricola, Piracicaba, v. 55, n. 2, p. 296-304, maio/ago. 1998.

FACHINELLO, J. C.; HOFFMANN, A.; NACHTIGAL, J. C.; KERSTEN, E.; FORTES, G. R. L. Propagação de plantas frutíferas de clima temperado. Pelotas: Ufpel, 1995. $179 \mathrm{p}$.

FACHINELLO, J. C.; KERSTEN, E.; MACHADO, A. A. Efeito do ácido indolbutírico no enraizamento de estacas lenhosas de pessegueiro cv. Diamante. Pesquisa Agropecuária Brasileira, Brasília, v. 17, n. 2, p. 247-252, fev. 1982.

FACHINELlO, J. C.; KERSTEN, E.; SILVEIRA JÚNIOR, P. Efeito do ácido indolbutírico na porcentagem de estacas lenhosas enraizadas e na obtenção de mudas de pessegueiro (Prunus persica (L.) Batsch). In: CONGRESSO BRASILEIRO DE FRUTICULTURA, 7., 1984, Florianópolis. Anais... Florianópolis: Sociedade Brasileira de Fruticultura, 1984. p. 1088-1096.

GARRIDO, G.; CANO, E. A.; ACOSTA, M.; SÁNCHEZBRAVO, J. Formation and growth of roots in carnation cuttings influence of storage period and auxin treatment. Scientia Horticulture, Amsterdam, v. 74, n. 3, p. 219 231, May 1998. 
HOFFMANN, A.; CHALFUN, N. N. J.; ANTUNES, L. E. C.; RAMOS, J. D.; PASQUAL, M.; SILVA, C. R. de R. e. Fruticultura comercial: propagação de plantas frutíferas. Lavras: Ufla/Faepe, 1996. 319 p.

HOFFMANN, A.; FACHINELLO, J. C.; SANTOS, A. M. dos. Propagação de mirtilo (Vaccinium ashei Reade) através de estacas. Pesquisa Agropecuária Brasileira, Brasília, v. 30, n. 2, p. 231-236, fev. 1995.

KAUNDAL, G. S.; KANWAR, J. S.; BRAR, S. S.; DEOL, I. S.; CHANANA, Y. R. Effect of growth regulators on the rhizogenesis of peach cultivars. Indian Journal of Horticulture, Bangalore, v. 50, n. 4, p. 318-326, 1993.

KERSTEN, E. Efeito do boro, zinco e ácido indolbutírico no enraizamento de estacas de dois cultivares de ameixeira (Prunus salicina, Lindl.). 1991. 109 f. Tese (Doutorado em Solos e Nutrição de Plantas) Escola Superior de Agricultura Luiz de Queiroz, Piracicaba.

KERSTEN, E.; TAVARES, W. S.; NACHTIGAL, J. C. Influência do ácido indolbutírico no enraizamento de estacas de ameixeira (Prunus salicina, Lindl.). Revista Brasileira de Fruticultura, Cruz das Almas, v. 16, n. 1, p. 215222, 1994.

LEONEL, S.; RODRIGUES, J. D.; CEREDA, E. Ação de fitorreguladores e ácido bórico em estacas de lichia (Litchi chinensis Sonn.). Científica, São Paulo, v. 22, n. 1, p. 105110, 1994.

MANUAL para produção de mudas de fruteiras. Pelotas: Ufpel/Embrapa-CNPFT, 1988. 123 p. Curso nacional sobre produção e inspeção de mudas de plantas frutíferas.

MATTIUZ, B.-H.; FACHINELLO, J. C. Enraizamento de estacas de Kiwi Acinidia deliciosa (A. Chev.) C. F. Liang e A. R. Ferguson var. Deliciosa. Pesquisa Agropecuária Brasileira, v. 31, n. 7, p. 503-508, jul. 1996.
MÉSEN, F.; NEWTON, A. C.; LEAKEY, R. R. B. Vegetative propagation of Cordia alliodora (Ruiz \& Pavon) Oken: the effects of IBA concentration, propagation medium and cutting origin. Forest Ecology and Management, Amsterdam, v. 92, n. 1/3, p. 45-54, May 1997.

ONO, E. O.; RODRIGUES, J. D. Aspectos da fisiologia do enraizamento de estacas caulinares. Botucatu: Unesp/Funep,1996. 83 p.

PACHECO, A. C.; CASTRO, P. R. C.; CLÓRIA, B. P. da. Aspectos anatômicos do enraizamento da videira muscadínia (Vitis rotundifolia Michx) através de alporquia. Scientia Agricola, Piracicaba, v. 55, n. 2, p. 210-217, maio/ ago. 1998.

PASINATO, V.; NACHTIGAL, J. C.; KERSTEN, E. Enraizamento de estacas lenhosas de cultivares de ameixeira (Prunus spp.), em condições de campo. Scientia Agricola, Piracicaba, v. 55, n. 2, p. 265-268, maio/ago. 1998.

ROSSAL, P. A. L.; KERSTEN, E.; CONTER, P. F. Estudo comparativo da evolução do nível de triptofano em ramos de ameixeira (Prunus salicina Lindl.). Scientia Agricola, Piracicaba, v. 54, n. 3, p. 174-177, set./dez. 1997.

SHARMA, S. D.; AIER, N. B. Seasonal rooting behaviour of cuttings of plum cultivars as influenced by IBA treatments. Scientia Horticulturae, Amsterdam, v. 40, n. 4, p. 297-303, Nov. 1989.

TONIETTO, A.; DUTRA, L. F.; KERSTEN, E. Influência do ácido indolbutírico e ethephon no enraizamento de estacas de pessegueiro (Prunus persica (L.) Batsch). Ciência Rural, Santa Maria, v. 27, n. 4, p. 567-569, out./ nov. 1997. 